\title{
In-situ Growth of BN Nanocages
}

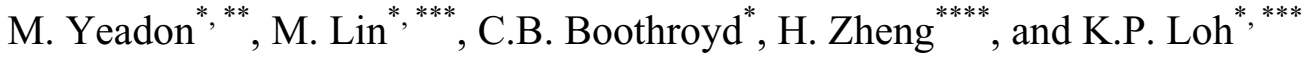 \\ *Institute of Materials Research and Engineering \\ ${ }^{* *}$ Dept of Materials Science, National University of Singapore, Singapore 119260 \\ *** Dept of Chemistry, National University of Singapore, Singapore 119260 \\ ${ }^{* * * *}$ Nanjing University, People's Republic of China
}

The growth of a variety of novel nanostructured carbon-based materials have been achieved in recent years following the discovery of fullerene molecules [1] and related nanostructures $[2,3]$. The formation of 'chains' of hollow spheres or 'nanocages' of C (e.g. $[4,5])$ has been observed, together with nanocages of hexagonal BN (e.g. [6]).

Using a modified high resolution ultrahigh vacuum transmission electron microscope we have succeeded in making direct observations of the formation of chains of h-BN nanocages by nitridation of $\mathrm{FeB}$ nanoparticles in $\mathrm{NH}_{3}$ under high vacuum conditions. Nanoparticles of $\mathrm{FeB}$ were prepared by ball-milling water-free $\mathrm{FeCl}_{3}$ and $\mathrm{NaBH}_{4}$ (1:3:3 in mole ratio) for $8 \mathrm{~h}$ followed by annealing in $\mathrm{Ar}$ at $500^{\circ} \mathrm{C}$ for $3 \mathrm{hr}$ [7] The FeB particles were then ultrasonicated in ethanol and cast onto Si supports, $6 \mathrm{~mm} \times 2 \mathrm{~mm} \times 0.2 \mathrm{~mm}$. One side of the support was uniaxially dimpled and chemically thinned to produce multiple perforations prior to casting of the particles.

Electron beam analysis of the particles revealed them to be $\sim 20-50 \mathrm{~nm}$ in diameter; electron energy loss spectroscopy (EELS) analysis revealed a clear B and C K-edges (figure 1, lower trace). The particles were then heated to $\sim 1000^{\circ} \mathrm{C}$ in a partial pressure of $\sim 5 \times 10^{-6}$ Torr $\mathrm{NH}_{3}$. The formation of thin, facetted shells around the particles was observed. The shells grew to a critical thickness at which point the FeB particle was spontaneously ejected from the shell. EELS analysis revealed the characteristic spectrum corresponding to hexagonal $\mathrm{BN}$, as shown in figure 1 (upper trace).

After further annealing, a second shell was observed to form around the FeB particle; at a similar critical thickness, the particle was again ejected. The process was repeated until a chain of $5 \mathrm{~h}-\mathrm{BN}$ cages had been formed, the last of which still contained the FeB particle. A bright field image of the sample after cooling to room temperature is shown in figure 2 .

The observations are consistent with a model for nanocage formation whereby the h-BN layers grow at the Fe-B interface by diffusion of $\mathrm{NH}_{3}$ through the growing $\mathrm{BN}$ layers. At a critical wall thickness, the particle is ejected from the nanocage, relieving compressive strain energy and creating a fresh FeB: vacuum interface. The formation of $\mathrm{BN}$ nanocages naturally depletes the B content until further nanocage growth ceases [8].

The authors thank the Singapore Agency for Science, Technology and Research (ASTAR) and Ministry of Education (MOE) for finanical support. 
[1] H.W. Kroto, J.R. Heath, S.C. O'Brien, R.F. Curl and R.E. Smalley, Nature 318 (1985) 162

[2] S. Iijima, Nature 354 (1991) 56

[3] T.W. Ebbesen and P.M. Ajayan, Nature 358 (1992) 220

[4] S. Seraphin, S. Wang, D. Zhou and J. Jiao, Chem. Phys. Lett. 228, 506 (1994)

[5] Y. Saitoh, T. Yoshikawa,M. Okuda, N. Fujimoto, K. Sumiyama, K. Suzuki, A. Kasuya and Y. Nishina, J. Phys. Chem. Solids 54, 1849 (1993)

[6] J. Cumings and A. Zettl, Chem. Phys. Lett. 316, 211 (2000)

[7] Z. Hu, Y. Fan and Y. Chen, Appl. Phys. A: Mater. Sci. Process. A68 (1999) 225

[8] M. Yeadon, M. Lin, C.B. Boothroyd, Z. Hu, and K.P. Loh, Appl. Phys. Lett., submitted

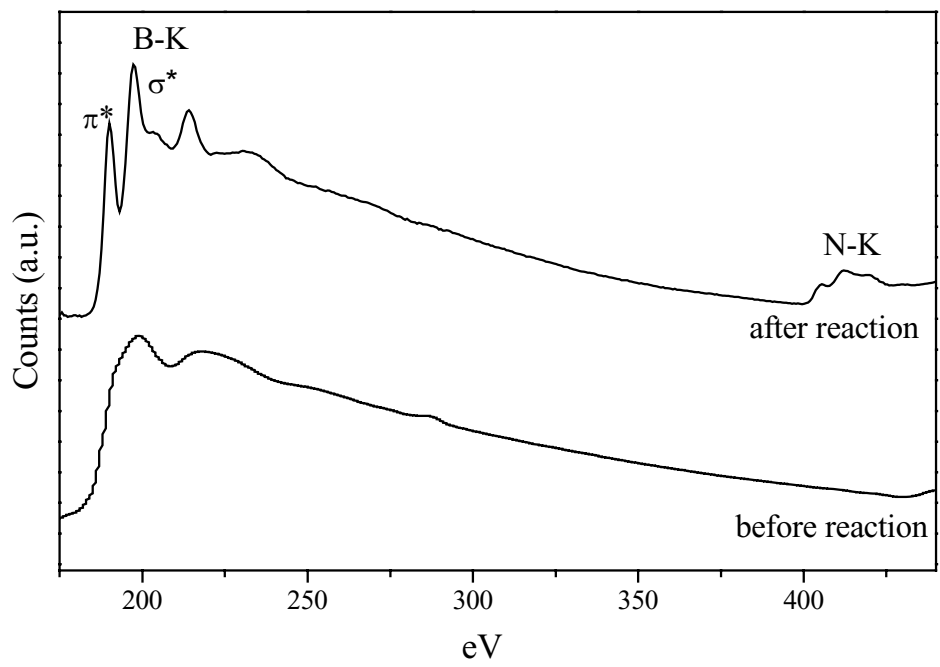

Figure 1. EELS spectra recorded form the sample before (lower trace) and after (upper trace) $\mathrm{NH} 3$ exposure at $1000^{\circ} \mathrm{C}$.

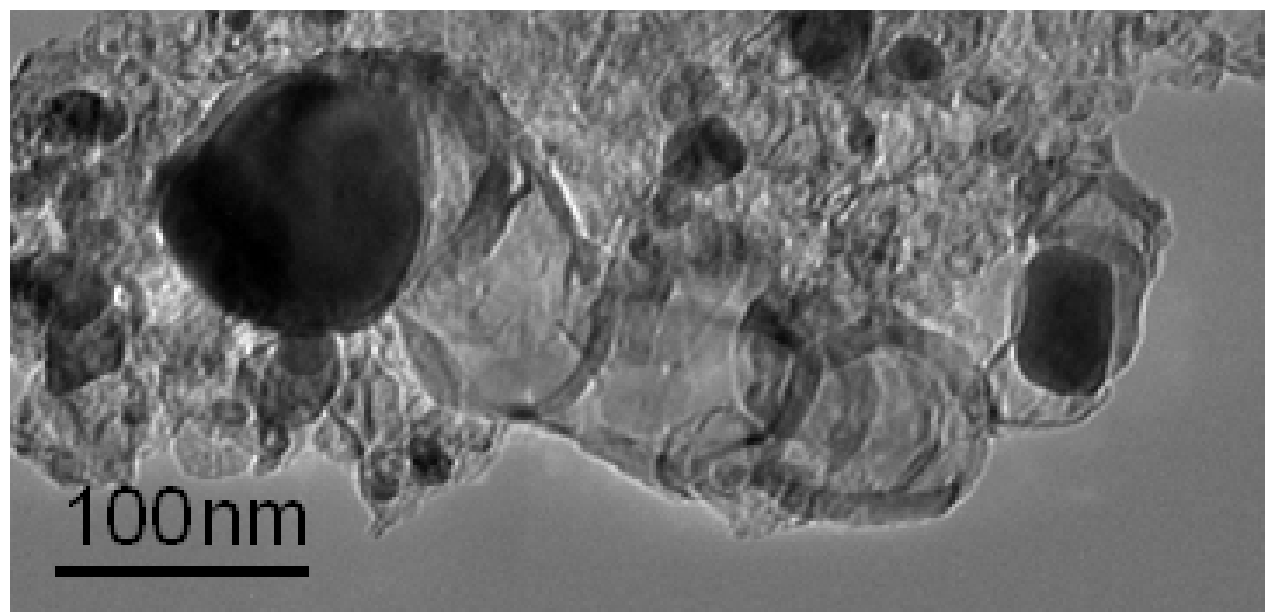

Figure 2. Bright field image of the nanocages formed by particle ejection; the $5^{\text {th }}$ nanocage (left) is still encapsulating the Fe-B particle. 